\title{
Assessment Criterion of Rigidity of Comb Electrodes Fingers of Microelectromechanical Converters
}

\author{
Boris Konoplev $^{1}$, Igor Lysenko ${ }^{1} \&$ Olga Ezhova ${ }^{1}$ \\ ${ }^{1}$ Department of Electronic Apparatuses Design, Institute of Nanotechnology, Electronics and Electronic \\ Equipment Engineering, Southern Federal University, Taganrog, Russia \\ Correspondence: Igor Lysenko, Department of Electronic Apparatuses Design, Institute of Nanotechnology, \\ Electronics and Electronic Equipment Engineering, Southern Federal University, Taganrog, Nekrasovsky st., 44, \\ GSP-17A, Russia. Tel: 786-3437-1603. E-mail: ielysenko@sfedu.ru
}

Received: October 16, 2015

Accepted: November 1, 2015

Online Published: December 24, 2015

doi:10.5539/mas.v10n2p52

URL: http://dx.doi.org/10.5539/mas.v10n2p52

\begin{abstract}
The assessment criterion of rigidity of comb electrodes fingers of the microelectromechanical converters is developed. The assessment criterion allows to estimate the maximum electrodes fingers length to decrease approach probability of snap-down effect. The modeling results have been analyzed. The estimations of the maximum length of electrodes fingers are obtained. The estimates depend on length of electrodes fingers overlapping and applied voltage. The dependences of the maximum length of comb electrodes fingers on length of their overlapping and applied voltage are showed. Since all of the electromechanical converters are reversible, i.e. the converters can work as electrostatic actuators, as well as capacitive displacement transducers. If certain conditions occurs, the capacitive displacement transducers will start to work like electrostatic actuators. The criteria allowed to define these invertibility condition can be obtained from the equilibrium equations of sensitive elements of micromechanical devices. The proposed assessment criterion of the maximum length of combs electrodes fingers can be used to design micromechanical devices.
\end{abstract}

Keywords: microelectromechanical system, electromechanical converters, model, modeling

\section{Introduction}

Microsystems technology emerged in the mid-eighties of last century at the junction of electronics, mechanics and optics. The microsystems technology remains one of the most dynamically developing scientific and technical sphere. Microelectromechanical systems (MEMS) are based on microsystems technology components. MEMS are devices with integrated in or on a solid body electronic and micromechanical structures. MEMS integration with optical components allows to allocate a separate class of components of the microsystems (Verner et al., 2007). This class is called microopticoelectromechanical systems. Basic elements of these systems are microelectromechanical converters including electrostatic actuators and capacitive displacement transducer (Verner, Malcev, Reznev, Saurov, \& Chaplygin, 2008).

Designing of electrostatic actuators and capacitive displacement transducer of micromechanical devices it is necessary to consider a number of effects that can cause snap-down effect of movable and fixed structures (Gridchin \& Dragunov, 2004).

\section{Problem Statement}

As stated in the principles of the micromechanical devices (Gridchin \& Dragunov, 2004; Lysenko, 2013; Raspopov, 2007) the reciprocating or rotary motion of movable elements is necessary for correct work. However, as shown in Figure 1, the electric field arises in cause the deflection voltage is applied between the movable and fixed electrodes of electromechanical converters. The electric field leads to a deviation of movable electrodes is not only along the $y$-axis, but also along the $\mathrm{x}$-axis and z-axis (Palaniapan, 2002). 


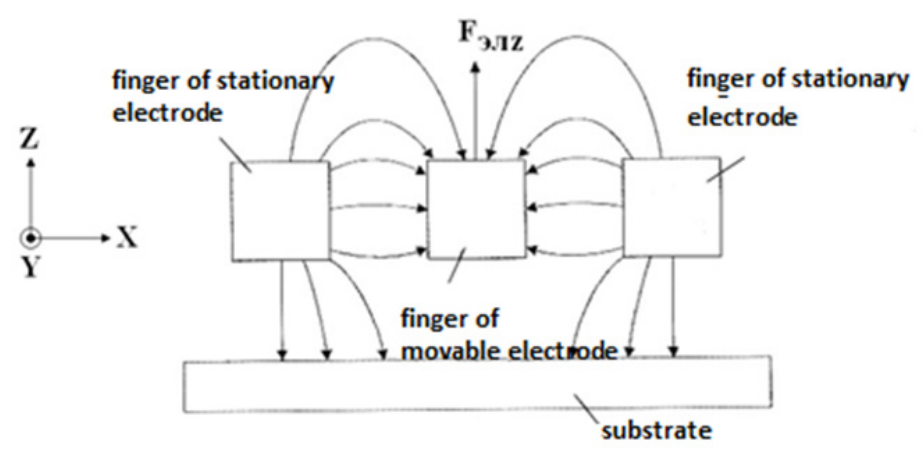

Figure 1. Scheme of interaction of combs electrodes of electrostatic actuator

The movable and fixed electrodes of electromechanical converters are manufactured in one structural layer, therefore it is possible to select the type of technological errors such as drift gap between the fingers (Palaniapan, 2002). The specified technological error leads to oscillations of moving elements under the action of the resulting electrostatic forces directed along the $\mathrm{x}$-axis. These oscillations are caused by tension/compression of beams of an elastic suspension of movable electrodes.

As shown in works (Palaniapan, 2002; Raspopov, 2007).the oscillation amplitude is tiny percent from the gap size between electrodes fingers and affects to emergence of snap-down effect slightly.

Besides, the electric field along $x$-axis would capable to cause snap-down effect of combs electrodes fingers of electromechanical converters, if fingers rigidity is insufficient, as shown in Figure 2 (Palaniapan, 2002).

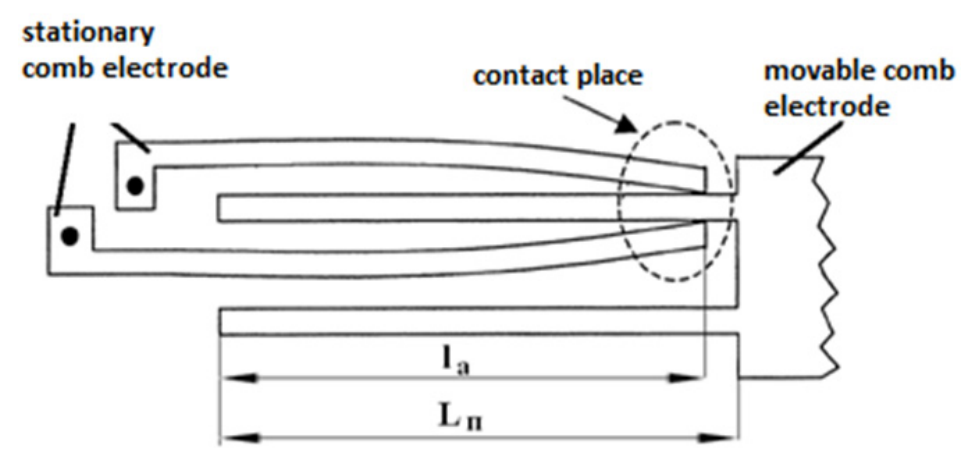

Figure 2. Electrostatic attraction of movable and fixed electrodes

Therefore designing the topology of electrostatic actuators and capacitive displacement transducers of micromechanical devices, it is necessary to consider the impact of this component of the electric field and introduce the criterion stiffness of the fingers of movable and fixed electrodes of electromechanical converters.

\section{Results and Discussion}

Based on expressions for calculation rigidity of cantilever beams and of electrostatic forces, generated planar electrostatic actuators (Raspopov, 2007; Pristupchik, 2015) the criterion of the maximum length of electrodes fingers of electromechanical converters are obtained:

$$
L=l_{a}+w g_{0} \sqrt[3]{\frac{E}{18 l_{a} \varepsilon \varepsilon_{0} U^{2}}},
$$

where $w$-width of the comb electrodes fingers; $l_{a}$-length of overlapping of the comb electrodes fingers; $g_{0}-$ distance between fingers of combs of movable and fixed electrodes; $E$-Young's modulus; $\varepsilon$-relative dielectric permittivity of an air-gap; $\varepsilon_{\mathbf{0}}$-an electric constant; U-voltage.

Figure 3-6 show the dependences of the maximum length of comb electrodes fingers on length of their overlapping 
and applied voltage.

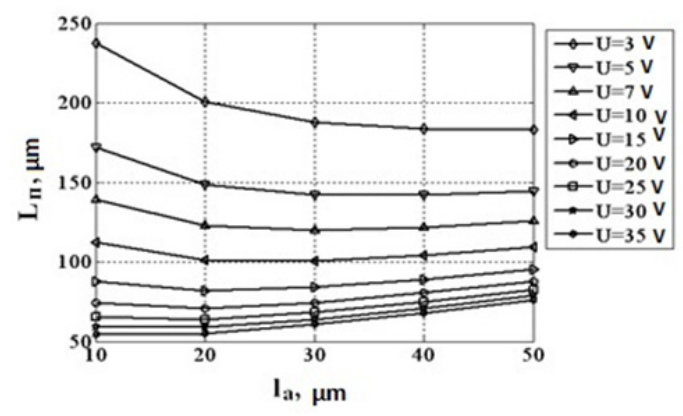

Figure 3. Dependence of the maximum length of combs electrodes fingers on length of their overlapping at $w=1$ $\mu \mathrm{m}, g_{0}=1 \mu \mathrm{m}$

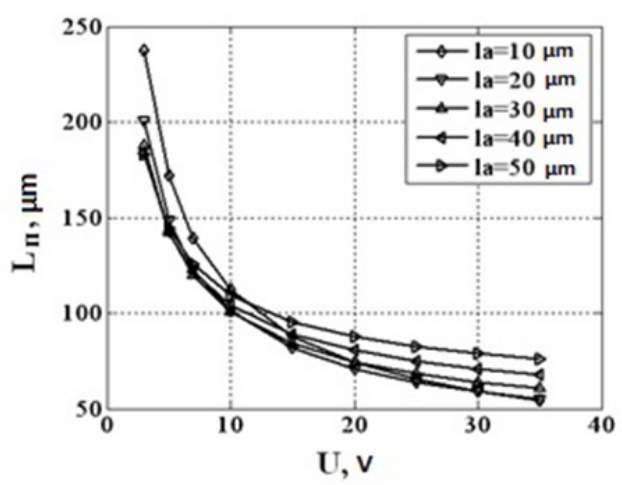

Figure 4. Dependence of the maximum length of combs electrodes fingers on applied voltage at $\mathrm{W}=1 \mu \mathrm{m}, g_{0}=$ $1 \mu \mathrm{m}$

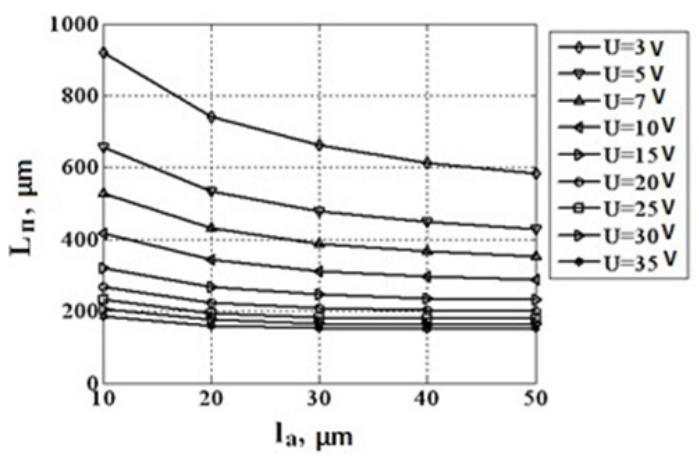

Figure 5. Dependence of the maximum length of combs electrodes fingers on length of their overlapping at $w=2$ $\mu \mathrm{m}, g_{0}=2 \mu \mathrm{m}$

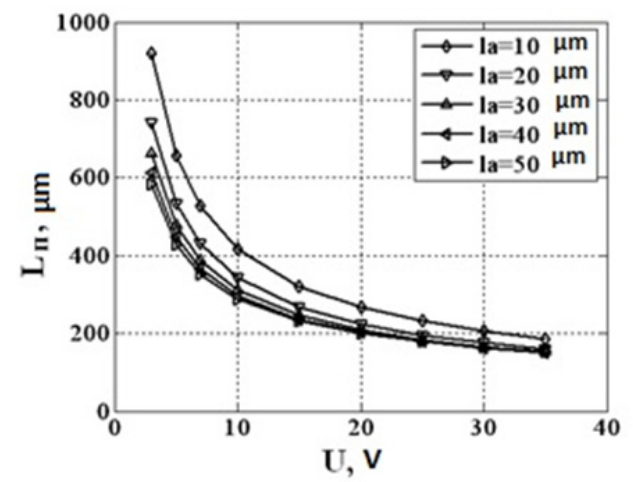


Figure 6. Dependence of the maximum length of combs electrodes fingers on applied voltage at $w=2 \mu \mathrm{m}, g_{0}=2$ $\mu \mathrm{m}$

The electrostatic actuators produce the electric field. The influence of the electric field along the z-axis causes levitation effect of moving structures of micromechanical gyroscope and, consequently, the existence of the quadrature error. The substrate is grounded to eliminate the levitation effect. As result there is asymmetric electric field. The field acts in the upper part of electrostatic actuators and can not cause oscillations with an amplitude affecting the quadrature error significantly. Additional fixed electrodes can be applied to eliminate the asymmetric electric field. The additional electrodes can be locate over comb structures movable and fixed electrodes (Palaniapan, 2002).

\section{Conclusions}

Since all of the electromechanical converters are reversible (Gridchin \& Dragunov, 2004; Raspopov, 2007), i.e., the converters can work as electrostatic actuators, as well as capacitive displacement transducers. If certain conditions occurs, the capacitive displacement transducers will start to work like electrostatic actuators. The criteria allowed to define these invertibility condition can be obtained from the equilibrium equations of sensitive elements of micromechanical devices.

The proposed assessment criterion of the maximum length of combs electrodes fingers can be used to design micromechanical devices.

\section{Acknowledgements}

This study was supported by the Ministry of Education and Science of Russia (project no. 14.575.21.0045, RFMEFI57514X0045).

\section{References}

Gridchin, V. A., \& Dragunov, V. P. (2004). Microsystems physics: Tutorial. Novosibirsk, NGTU publishing house.

Lysenko, I. E. (2013). Modeling of the Micromachined Angular Rate and Linear Acceleration Sensor LL-Type with Redirect of Drive and Sense Axes. World Applied Sciences Journal, 27, 759-762.

Palaniapan, M. (2002). Integrated surface micromachined frame microgyroscopes. Berkeley, University of California.

Pristupchik, N., Konoplev, B., Kulikova, I., Klunnikova, Y., \& Lysenko, I. (2015). Quantum Tunneling in Mesoscopic Electromechanical Transducers. Modern Applied Science, 9, 20-29.

Raspopov, V. J. (2007). Micromechanical devices. Moscow, Mashinostroenie.

Verner, V. D., Ivanov, A. A., Kolomenskaja, N. G., Luchinin, V. V., Malcev, P. P., Popova, I. V., ... Telec, V. A. (2007). Products microsystem technology-the basic concepts and terms. Journal of Nano and Microsystem Technique, 12, 2-5.

Verner, V. D., Malcev, P. P., Reznev, A. A., Saurov, A. N., \& Chaplygin, J. A. (2008). Modern trends in microsystem technology. Journal of Nano and Microsystem Technique, 8, 2-6.

\section{Copyrights}

Copyright for this article is retained by the author(s), with first publication rights granted to the journal.

This is an open-access article distributed under the terms and conditions of the Creative Commons Attribution license (http://creativecommons.org/licenses/by/3.0/). 